\title{
Impact of a Clinical Pharmacist in the General Hospital: An Egyptian Trial
}

\author{
Nirmeen A. Sabry, Maggie M. Abbassi* \\ Clinical Pharmacy Department, Faculty of Pharmacy, Cairo University, Cairo, Egypt \\ Email: ${ }^{*}$ maggieabbassi@gmail.com
}

Received 22 February 2014; revised 15 May 2014; accepted 4 June 2014

Copyright (C) 2014 by authors and Scientific Research Publishing Inc.

This work is licensed under the Creative Commons Attribution International License (CC BY). http://creativecommons.org/licenses/by/4.0/

c) (7) Open Access

\begin{abstract}
Medication review aims at optimizing the impact of medications while minimizing their related problems. Drug-related problems have never been properly addressed in Egyptian hospitals. The purpose of this paper is to record and compare the prevalence and types of medication related problems, the interventions provided by the clinical pharmacists and how physicians responded along with making recommendations for error prevention. This prospective study was conducted between June 2012 and December 2012, at an Egyptian general hospital. Five trained pharmacists recorded patient-specific medication related recommendations and completed quality-of-care interventions. The average number of audited doses was $81 \%$ of those prescribed. The most prevalent medication problem was prescribing errors followed by administration errors. A total of 20 patients experienced adverse drug events. The greatest error rates across the seven months were observed in the ICU and cardiology units. Numbers of interventions offered by the pharmacists ranged from 241, to 519 per month. Nurses accepted all the interventions introduced by the pharmacists aimed at reducing administration errors while physicians' resistance rates had an average of $21 \%$. This study showed a positive influence of the pharmacist-led medication review in reducing potential drug-related problems in an Egyptian secondary care where the hospital under study implemented new measures to minimize drug related problems according to the findings of the trained pharmacists.
\end{abstract}

\section{Keywords}

Egypt, Medication Review, Intervention, Drug Related Problems

\section{Introduction}

Patient safety is a main goal in any treatment protocol. Drugs are not licensed worldwide until they are proven to

"Corresponding author. 
be safe and efficacious. However, drug related problems (DRPs) represent a worldwide concern. A DRP can be defined as "A circumstance that involves a patient's drug treatment that actually, or potentially, interferes with the achievement of an optimal outcome" [1]. This can include any stage of drug use starting from the prescribing process, all through dispensing, administration and then possible adverse events. The frequency of DRPs is ranging in the literature from $1.7 \%$ to $59 \%$ according to definitions of problems studied and the setting in which the study was conducted [2]. It is being documented that, DRPs are one of the reasons for increasing the length of hospital stays and overall treatment costs [2]. A Danish medication report found that, 8.7\% of medication errors can result in death [3]. The interest in DRPs was greatly stimulated by the report of the Institute of Medicine "To err is human" [4], which emphasized the fact that, even though errors are inevitable yet, they can be minimized to build a safer healthcare system.

Although medication review process is a routine pharmacy practice in many countries in Europe and the United States for more than 30 years now, this practice is recently introduced and is developing in the Middle East countries including Egypt.

Only few studies were done in Egyptian hospitals to identify DRPs and were performed on a small scale inside one unit of a hospital: Intensive care unit, pediatric intensive care unit and in an obstetric emergency ward [5]-[7]. Even though, DRPs have been studied worldwide, extrapolating international solutions to local settings might not always be applicable. One of the main reasons for the lack of monitoring DRPs in Egyptian hospitals is that, clinical pharmacy practice in Egyptian hospitals has been only recently introduced and implemented in only few of the hospitals.

Increasing interest in implementing this practice has recently stemmed after the Egyptian Ministry of Health mandated that, all hospitals must implement clinical pharmacy practices before July 2013. An important reason for the delay in implementing a significant role for the pharmacists within Egyptian hospitals is the physician's resistance to pharmacist's role.

With the implementation of this practice in several hospitals in Egypt, the aim of this study arouse, which was recording, and comparing the prevalence, and types of medication related problems, the interventions provided by the clinical pharmacists and how physicians responded in one of the trial Egyptian hospitals along with making recommendations for error prevention, emphasizing the role of clinical pharmacists in an Egyptian setting.

\section{Methods and Materials}

This work was conducted after an approval and permission from the Ethics Committee of the Faculty of Pharmacy, Cairo University. Authors have no conflicts of interest with regard to the data produced. A cross-sectional evaluation of medication review improvement service was conducted in a private Egyptian general hospital (150 beds) of multiple specialties. All the patients admitted to the hospital were subjected to a thorough medication review process during their hospital stay. This service was part of a bigger quality improvement project that was designed for all the hospital services. Before applying this service, the pharmacist's role was limited to receiving physicians' orders, screening it for medication availability and dispensing the medications. The first step in launching the service was a careful selection and recruitment of clinical pharmacists to carry out the medication review process. The selected pharmacists were trained to review patient files, identify DRPs, communicate with other healthcare professions and document valid data. The training process was done by the principle investigator who has already received her training in one of the teaching hospitals in the United Kingdom, over a period of 4 years.

\subsection{Data Collection}

The characteristics of the hospital and wards are presented in Table 1. Because this was a service evaluation, no sample size was decided. Instead, a 7 month period was selected starting from the June 2012 which was the first month of launching the service. During this period all the reviewing pharmacists were requested to review as many patients as they can and to record any identified problems together with any suggested interventions. All the reviewed patients were considered the study sample size and the percentages of patients reviewed were calculated.

Patient data collection sheet was specially designed to include all the patients' relevant data including demographics, reason(s) of hospital admission, co-morbidities, prescribed medication during hospital stay, and all the relevant biochemical data done during the hospital stay. 
Table 1. Characteristics of hospital and wards being studied.

\begin{tabular}{ll}
\hline Hospital type & Private \\
\hline Beds & 150 \\
Inpatient prescribing & Paper based and computer based \\
Discharge prescribing & Paper based and computer based \\
Number of Wards & 8 general wards served by 15 specialty + \\
Pharmacist availability on & 5 critical areas \\
ward & 9 am-3 pm shift \\
\hline
\end{tabular}

Pharmacists documented details of DRPs identified, the number of doses administered (or omitted) to the patient in the hospital before the problem was identified, together with any proposed intervention(s) to correct the problem and the response to the proposed interventions. DRPs were detected through both file review and clinical round attendance. The outcome measures were the rates and types of DRPs, the types and frequencies of recommendations proposed by pharmacists and the number of the recommendations, which were accepted or implemented by the treating physicians.

A carefully designed DRPs list was specially created to be used in the hospital as a guidance to record all the identified problems in a structured way. The problems were mutually exclusive and included: indication with no prescribed medication [8], medications with no valid indication [8], overdose: accidental or intentional use of a drug or medicine in an amount that is higher than is normally used [8] [9], underdose: accidental or intentional use of a drug or medicine in an amount that is less than is normally used [8] [9], drug-drug interaction (Using online Lexi-interact $\left.{ }^{\circledR}\right)$ : occurring whenever the effects of one drug are modified in or on the body by the prior or concurrent administration of another pharmacologically active substance. Only those interactions required dose modification, changing schedules, or avoiding combination were recorded [8] [9], adverse drug events: any adverse, noxious or harmful event associated with the use of a drug in humans, whether or not considered drug related, as defined by The United States Food and Drug Administration (FDA) [10].

Lack of therapeutic drug monitoring (TDM) and/or resultant need of adjustment of drug doses, drug duplication: the use of multiple agents from the same chemical family or therapeutic class [9], contraindication: a condition or factor that serves as a reason to withhold a certain medical treatment, therapeutic failure when the expected drug effects do not occur following a prescribed pharmacological treatment, including any clinical event that could be related to a low prescribed dose [9]. Also, nursing administration problems were recorded. This was defined as any deviation from the physician's medication order as written on the patient's chart [11], including timing problems, missing doses as observed from the administration sheet and after confirming with the nurse, an extra dose as observed from the quantities of unit doses remaining in the patient's tray, or wrong infusion flow rate. Finally, prescribing errors adopted from Anderson 2003 [9] and described as drug repetition, unclear writing (abbreviation, concentration, frequency), wrong interpretation of the culture and sensitivity, incomplete PRN medication order, wrong dose, dosage form, or wrong route of administration, and wrong timing of medication administration were also documented and corrected whenever it was possible.

\subsection{Interventions}

Intervention was defined as "any kind of measure taken by the pharmacy staff members to solve a detected drug-related problem" [9]. All the interventions recorded were proposed by the clinical pharmacy team on the level of the prescriber or on the level of nurses. None were done on the level of patients or drug. On the prescriber level, the prescribers were either informed only, intervention proposed, approved by prescriber, or intervention proposed, but not approved by prescriber.

\subsection{Data Analysis}

Prevalence of medication problems, breakdown by specialty, interventions done by the pharmacists and rate of resistance to pharmacist interventions were calculated. Ninety five percent confidence intervals $(95 \% \mathrm{CI})$ were calculated when possible. Prevalence of medication problems was calculated as number of error doses as a fraction of total audited doses. Percentage of problems per specialty was calculated as number of error doses per 
specialty as a fraction of total error doses for this month. Interventions done by the pharmacists per specialty were counted; the resistance rate was calculated as the number of times the intervention was refused by the physician or if the physician could not be reached as a fraction of the total number of interventions per specialty. In case of accepting the intervention, the doses taken or missed before the error was corrected were also counted.

Although demographic data (gender, weight, height) were collected for the accuracy of medication review process, demographic data were not included in the analysis process as the aim was service focused rather than patient focused.

\section{Results}

\subsection{Frequencies and Types of Medication Problems}

Because of the nature of the hospital and being a general hospital, it was inaccurate to describe the identified problems per patient due to major variability in the number of medications prescribed for each patient. Instead, the problems were recorded based on the total dispensed doses. The number of medications taken by patients ranged from 1 to 20 during the seven months. The total numbers of prescribed doses in the hospital were 52887, 48412, 48317, 52359, 44896, 44541 and 49622 for the seven months period starting in June, respectively, in 11 wards including surgical and intensive care units (ICUs). Total audited doses by the pharmacists were 43072 (81\%), 33096 (68\%), 36509 (76\%), 37129 (71\%), 35866 (80\%), 43240 (97\%), and 48749 (98\%) with an average rate of $81 \%$.

The number of doses found to have problems were 1197 (2.8\% (2.6 to 2.9)), 856 (2.6\% (2.4 to 2.8)), 624 (1.7\% (1.6 to 1.8$)$ ), 1248 (3.4\% (3.2 to 3.6)), 1489 (4.2\%( 4 to 4.4)), 1473 (3.4\% (3.2 to 3.6)) and 902 (1.9\% (1.7 to 2 )) for the seven consecutive months respectively with an average rate of $2.8 \%$.

The average percentages of each problem over the seven months are shown in Figure 1. The most prevalent medication problem in the seven months was prescribing errors followed by administration errors. Medication

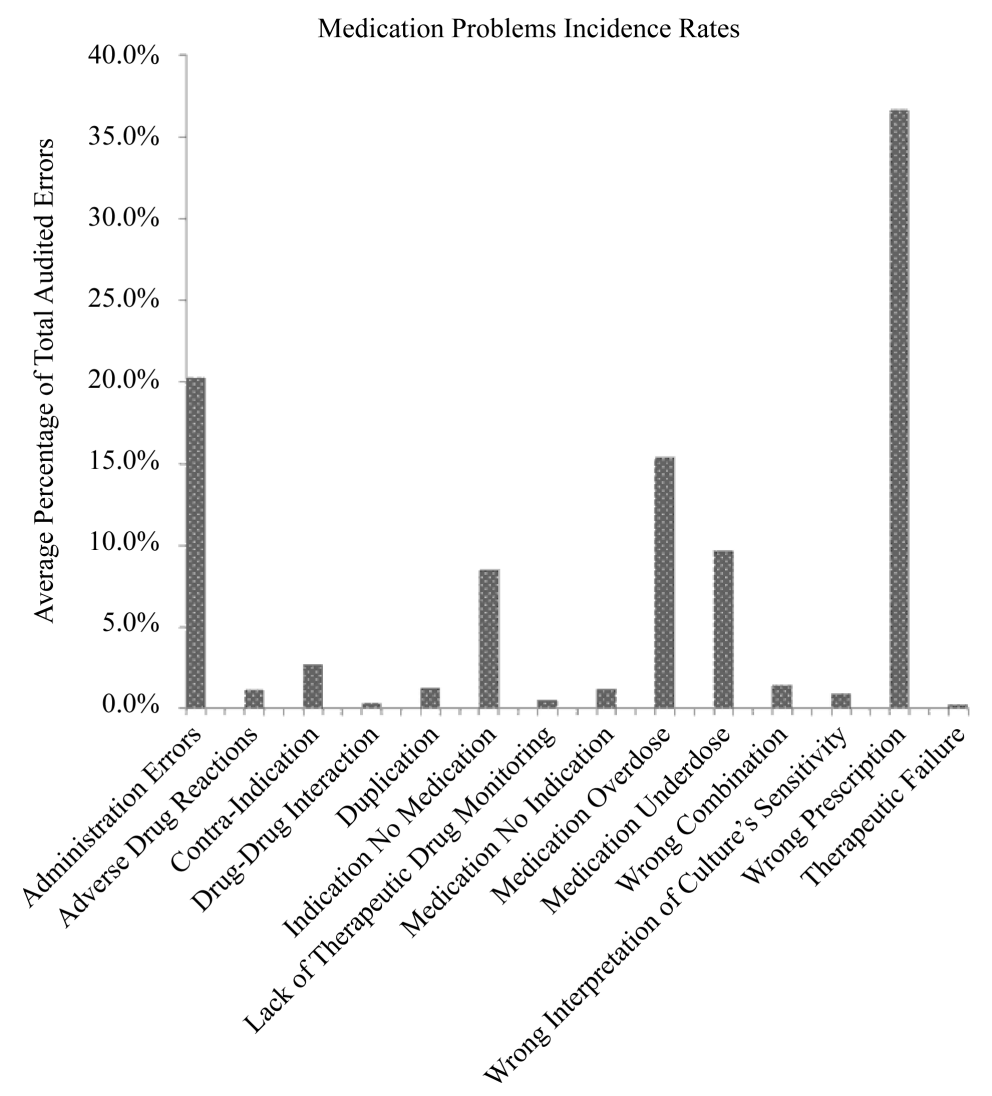

Figure 1. Average incidence of different identified medication problems in seven months period. 
overdose was the third reported problem especially in patients with renal impairment who were not subjected to dose adjustment according to their kidney function. The 95\% confidence intervals are shown in Table 2.

During the study period, a total of 20 (92 doses) patients experienced adverse drug events. One of them only was recorded as allergic and the rest were non-allergic events. Thirteen (70 doses) of the recorded events were described as serious events according to the FDA definitions [12]. These events resulted in death or serious events, and required urgent intervention including ICU admission, discontinuing medication, treatment change, or extra monitoring. Due to confidentiality issues, it was quite difficult to obtain total death cases recorded in the hospital during the evaluation period, which in turn made it difficult to calculate the medication related mortalities.

\subsection{Interventions by Pharmacists}

Pharmacists were requested to intervene whenever a problem was identified. The interventions permitted by the hospital authorities were either informing the nurse for any administration problems or the physician for all other types of problems. Total numbers of interventions offered by the pharmacists were 241, 278, 183, 421, 388, 519 and 405 for the seven months with resistance rates $21 \%$ (16\% to $27 \%$ ), $33 \%$ (27\% to 39\%), 31\% (25\% to

Table 2. Percentage of doses of each type of problem from total error doses per month (95\% confidence interval).

\begin{tabular}{|c|c|c|c|c|c|c|c|}
\hline $\begin{array}{c}\text { Types of } \\
\text { Identified } \\
\text { Problems (\%) }\end{array}$ & June & July & August & September & October & November & December \\
\hline \multirow{2}{*}{$\begin{array}{c}\text { Administration } \\
\text { Errors }\end{array}$} & $7.9 \%$ & $14.6 \%$ & $16.0 \%$ & $29.3 \%$ & $21.8 \%$ & $28.4 \%$ & $23.8 \%$ \\
\hline & $(6.4 \%-9.6 \%)$ & $(12.3 \%-17.2 \%)$ & $(13.3 \%-19.2 \%)$ & $(26.8 \%-32.0 \%)$ & $(19.7 \%-24.0 \%)$ & $(26.1 \%-50.8 \%)$ & $(21.1 \%-26.8 \%)$ \\
\hline \multirow{2}{*}{$\begin{array}{c}\text { Adverse Drug } \\
\text { Reaction }\end{array}$} & $2.8 \%$ & $0.5 \%$ & $0.8 \%$ & $0.8 \%$ & $0.1 \%$ & $2.3 \%$ & $0.9 \%$ \\
\hline & $(2.0 \%-4.0 \%)$ & $(0.1 \%-1.3 \%)$ & $(0.3 \%-2.0 \%)$ & $(0.4 \%-1.5 \%)$ & N/A & $(1.6 \%-3.2 \%)$ & $(0.4 \%-1.8 \%)$ \\
\hline \multirow{2}{*}{$\begin{array}{c}\text { Contra } \\
\text { Indication }\end{array}$} & $3.1 \%$ & $2.2 \%$ & $3.8 \%$ & $1.0 \%$ & $0.8 \%$ & $3.3 \%$ & $5.0 \%$ \\
\hline & $(2.2 \%-4.3 \%)$ & $(1.4 \%-3.5 \%)$ & $(2.5 \%-5.8 \%)$ & $(0.6 \%-1.8 \%)$ & $(0.4 \%-1.4 \%)$ & $(2.4 \%-4.3 \%)$ & $(3.7 \%-6.7 \%)$ \\
\hline \multirow{2}{*}{$\begin{array}{l}\text { Drug-Drug } \\
\text { Interaction }\end{array}$} & $0.3 \%$ & $0.5 \%$ & $0.8 \%$ & $0.4 \%$ & $0.0 \%$ & $0.2 \%$ & $0.6 \%$ \\
\hline & $(0.1 \%-0.9 \%)$ & $(0.1 \%-1.3 \%)$ & $(0.3 \%-2.0 \%)$ & $(0.1 \%-1.0 \%)$ & N/A & $(0.1 \%-0.6 \%)$ & $(0.2 \%-1.4 \%)$ \\
\hline \multirow{2}{*}{ Duplication } & $4.1 \%$ & $0.8 \%$ & $0.3 \%$ & $0.7 \%$ & $0.3 \%$ & $0.6 \%$ & $1.9 \%$ \\
\hline & $(3.1 \%-5.4 \%)$ & $(0.4 \%-1.8 \%)$ & $(0.1 \%-1.3 \%)$ & $(0.4 \%-1.4 \%)$ & $(0.1 \%-0.8 \%)$ & $(0.3 \%-1.2 \%)$ & $(1.1 \%-3.1 \%)$ \\
\hline \multirow{2}{*}{$\begin{array}{l}\text { Indication No } \\
\text { Medication }\end{array}$} & $11.5 \%$ & $10.4 \%$ & $10.4 \%$ & $6.5 \%$ & $4.1 \%$ & $8.3 \%$ & $8.6 \%$ \\
\hline & $(9.7 \%-13.4 \%)$ & $(8.5 \%-12.7 \%)$ & $(8.2 \%-13.1 \%)$ & $(5.2 \%-8.0 \%)$ & $(3.2 \%-5.3 \%)$ & $(6.9 \%-9.8 \%)$ & $(6.9 \%-10.7 \%)$ \\
\hline \multirow{2}{*}{$\begin{array}{c}\text { Lack of } \\
\text { Therapeutic } \\
\text { Drug Monitoring }\end{array}$} & $0.2 \%$ & $0.7 \%$ & $1.9 \%$ & $0.2 \%$ & $0.6 \%$ & $0.2 \%$ & $0.2 \%$ \\
\hline & $(0.0 \%-0.7 \%)$ & $(0.3 \%-1.6 \%)$ & $(1.0 \%-3.4 \%)$ & $(0.1 \%-0.8 \%)$ & $(0.3 \%-1.2 \%)$ & $(0.1 \%-0.6 \%)$ & $(0.0 \%-0.9 \%)$ \\
\hline \multirow{2}{*}{$\begin{array}{l}\text { Medication No } \\
\text { Indication }\end{array}$} & $1.2 \%$ & $0.4 \%$ & $0.6 \%$ & $0.7 \%$ & $0.9 \%$ & $1.6 \%$ & $3.2 \%$ \\
\hline & $(0.7 \%-2.0 \%)$ & $(0.1 \%-1.1 \%)$ & $(0.2 \%-1.7 \%)$ & $(0.4 \%-1.4 \%)$ & $(0.5 \%-1.5 \%)$ & $(1.0 \%-2.4 \%)$ & $(2.2 \%-4.6 \%)$ \\
\hline \multirow{2}{*}{$\begin{array}{l}\text { Medication } \\
\text { Overdose }\end{array}$} & $12.0 \%$ & $19.4 \%$ & $20.0 \%$ & $19.3 \%$ & $9.9 \%$ & $12.0 \%$ & $15.3 \%$ \\
\hline & $(10.2 \%-14.0 \%)$ & $(16.8 \%-22.2 \%)$ & $(17.0 \%-23.4 \%)$ & $17.2 \%-21.6 \%)$ & $(8.4 \%-11.5 \%)$ & $(10.4 \%-13.8 \%)$ & $(13.0 \%-17.9 \%)$ \\
\hline \multirow{2}{*}{$\begin{array}{l}\text { Medication } \\
\text { Underdose }\end{array}$} & $9.2 \%$ & $7.0 \%$ & $7.5 \%$ & $6.7 \%$ & $9.3 \%$ & $13.0 \%$ & $15.0 \%$ \\
\hline & $(7.6 \%-11.0 \%)$ & $(5.4 \%-9.0 \%)$ & $(5.6 \%-10.0 \%)$ & $(5.4 \%-8.2 \%)$ & $(7.9 \%-11.0 \%)$ & $(11.4 \%-14.9 \%)$ & $(12.7 \%-17.5 \%)$ \\
\hline \multirow{2}{*}{$\begin{array}{c}\text { Wrong } \\
\text { Combination }\end{array}$} & N/A & $1.3 \%$ & $2.1 \%$ & $1.0 \%$ & $2.9 \%$ & $1.3 \%$ & $0.0 \%$ \\
\hline & N/A & $(0.7 \%-0.7 \%)$ & $(1.2 \%-3.6 \%)$ & $(0.6 \%-1.8 \%)$ & $(2.1 \%-3.9 \%)$ & $(0.8 \%-2.0 \%)$ & N/A \\
\hline \multirow{2}{*}{$\begin{array}{c}\text { Wrong } \\
\text { Interpretation } \\
\text { of Culture's }\end{array}$} & N/A & $1.2 \%$ & N/A & $1.0 \%$ & N/A & $0.5 \%$ & $1.1 \%$ \\
\hline & N/A & $(0.6 \%-2.2 \%)$ & N/A & $(0.5 \%-1.7 \%)$ & N/A & $(0.2 \%-1.0 \%)$ & $(0.6 \%-2.1 \%)$ \\
\hline \multirow{2}{*}{$\begin{array}{l}\text { Prescribing } \\
\text { Errors }\end{array}$} & $47.7 \%$ & $41.1 \%$ & $35.6 \%$ & $32.3 \%$ & $48.8 \%$ & $27.7 \%$ & $23.6 \%$ \\
\hline & $(44.8 \%-50.6 \%)$ & $(37.8 \%-44.5 \%$ & $31.8 \%-39.5 \%$ & $29.7 \%-35.0 \%)$ & $(46.2 \%-51.3 \%)$ & $(25.4 \%-30.1 \%)$ & $(20.9 \%-26.6 \%)$ \\
\hline \multirow{2}{*}{$\begin{array}{c}\text { Therapeutic } \\
\text { Failure }\end{array}$} & $0.1 \%$ & N/A & N/A & N/A & N/A & $0.4 \%$ & $0.2 \%$ \\
\hline & $(0.0 \%-0.5 \%)$ & N/A & N/A & N/A & N/A & $(0.2 \%-0.9 \%)$ & $(0.0 \%-0.9 \%)$ \\
\hline
\end{tabular}


$39 \%), 16 \%$ (12\% to $20 \%$ ), $15 \%$ (12\% to $19 \%), 21 \%$ (18\% to $25 \%$ ) and $20 \%$ (16\% to $24 \%$ ) respectively with an average resistance or rejection of the proposed intervention of $21 \%$. When it comes to the response of the healthcare provider, the nurses always complied with the pharmacist recommendations while the resistance always came from physicians. Antibiotic, sedative/analgesic agents, cardiovascular, and gastrointestinal agents were the most common classes of medications in which dosing interventions were performed.

The number of problem doses per specialty was counted and the error rates were calculated. The average error rate per specialty is shown in Figure 2. The greatest error rates across the seven months were observed in the ICU units (average of 22\%) and cardiology units (average of 22\%). Internal medicine and surgery showed also high rates of problems (average of $10 \%$ and $9 \%$ respectively) compared to other specialties.

The breakdown of interventions per specialty and the resistance rates per specialty are shown in Table 3. The reported interventions were counted per medication problem not per dose. The 95\% confidence intervals were too wide and not statistically meaningful due to the low number of observations when broken down by month and specialty. Cardiology, ICU and internal medicine specialties had the highest number of interventions. Resistance rates were relatively high in these specialties as well as in surgery ward. Even though resistance by physicians to pharmacist interventions seemed to decrease across the study period or did not change for most of the specialties, it appeared to increase in the Internal medicine and ICU departments.

\section{Discussion}

This study is the first study to describe the role of clinical pharmacists as a hospital improvement service in detecting, avoiding, and correcting DRPs in an Egyptian general hospital for a period of seven months. This study records the pharmacists' interventions in the secondary care setting and the measures taken by the hospital as a result of the pharmacists' review. Pharmacists were effectively able to intervene and correct all audited administration errors while physicians especially consultants were more resistant to interventions.

The percentage of doses reviewed increased along the study except for August and September. Ramadan - the fasting month — came during this period and could be the reason behind this decline, where the daily working hours decreased from 6 hours daily to four hours only. The pharmacists were capable of auditing 98\% of all the prescribed doses in the hospital by the seventh month. The majority of DRPs in this study were avoidable as previously documented [13]. Seventy doses only were behind serious events all over the study period.

Critical care areas had the highest percentage of recorded DRPs. This could be attributed to the presence of

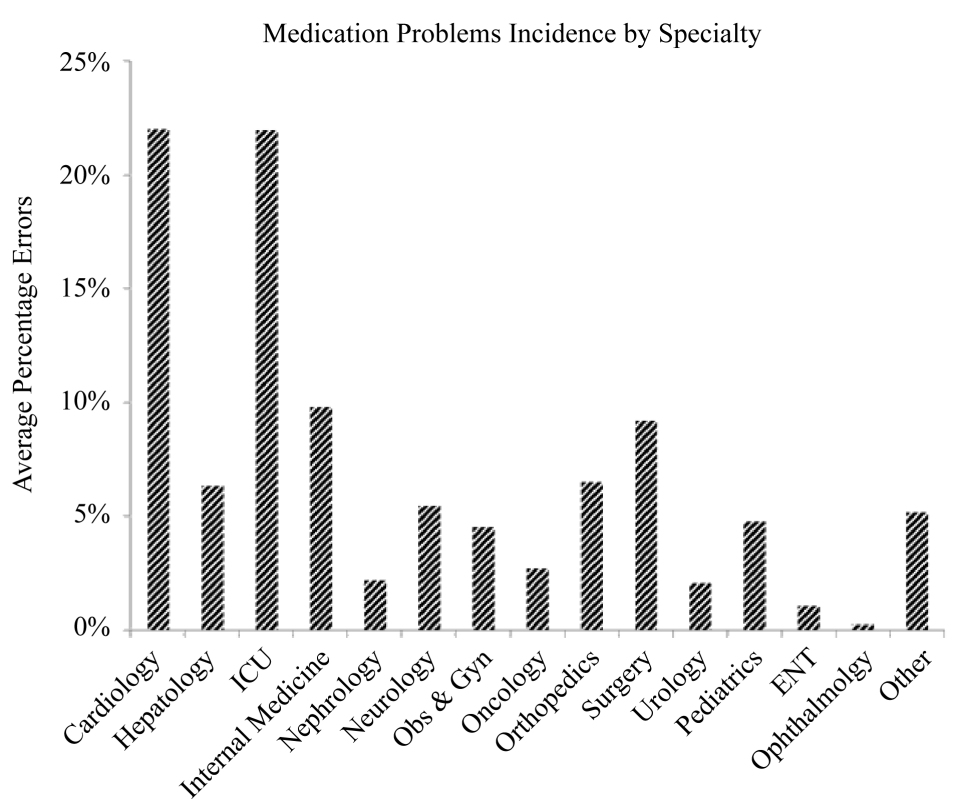

Figure 2. Medication problems incidence per specialty averaged over a period of seven months (ICU: Intensive Care Units, Obs \& Gyn: Obstetrics and Gynecology, ENT: Ear, nose and throat). 
Table 3. Number of interventions done by pharmacists per specialty per month, number of doses affected by positive interventions and resistance rate to pharmacist interventions divided by specialty.

\begin{tabular}{|c|c|c|c|c|c|c|c|c|}
\hline Specialty & & June & July & August & September & October & November & December \\
\hline \multicolumn{9}{|l|}{ Cardiology } \\
\hline & Number of interventions & 45 & 62 & 41 & 44 & 79 & 129 & 98 \\
\hline & Number of affected doses & 104 & 160 & 93 & 151 & 279 & 217 & 158 \\
\hline & Resistance rate & $33 \%$ & $31 \%$ & $24 \%$ & $18 \%$ & $23 \%$ & $31 \%$ & $23 \%$ \\
\hline \multicolumn{9}{|l|}{ Hepatology } \\
\hline & Number of interventions & 10 & 14 & 5 & 18 & 11 & 36 & 24 \\
\hline & Number of affected doses & 67 & 22 & 17 & 103 & 49 & 111 & 47 \\
\hline & Resistance rate & $20 \%$ & $50 \%$ & $0 \%$ & $17 \%$ & $0 \%$ & $11 \%$ & $21 \%$ \\
\hline \multicolumn{9}{|l|}{ ICU } \\
\hline & Number of interventions & 73 & 58 & 58 & 219 & 87 & 99 & 85 \\
\hline & Number of affected doses & 267 & 65 & 109 & 526 & 259 & 163 & 88 \\
\hline & Resistance rate & $14 \%$ & $29 \%$ & $43 \%$ & $16 \%$ & $14 \%$ & $16 \%$ & $28 \%$ \\
\hline \multicolumn{9}{|c|}{ Internal Medicine } \\
\hline & Number of interventions & 44 & 36 & 18 & 32 & 26 & 69 & 27 \\
\hline & Number of affected doses & 136 & 76 & 32 & 63 & 97 & 146 & 41 \\
\hline & Resistance rate & $16 \%$ & $33 \%$ & $33 \%$ & $16 \%$ & $15 \%$ & $20 \%$ & $30 \%$ \\
\hline \multicolumn{9}{|l|}{ Nephrology } \\
\hline & Number of interventions & 5 & 8 & 3 & 9 & 15 & 8 & 5 \\
\hline & Number of affected doses & 3 & 8 & 0 & 43 & 47 & 13 & 15 \\
\hline & Resistance rate & $60 \%$ & $50 \%$ & $8 \%$ & $0 \%$ & $20 \%$ & $25 \%$ & $40 \%$ \\
\hline \multicolumn{9}{|l|}{ Neurology } \\
\hline & Number of interventions & 7 & 21 & 13 & 8 & 20 & 22 & 21 \\
\hline & Number of affected doses & 2 & 70 & 25 & 35 & 69 & 61 & 38 \\
\hline & Resistance rate & $71 \%$ & $14 \%$ & $23 \%$ & $25 \%$ & $10 \%$ & $23 \%$ & $29 \%$ \\
\hline \multicolumn{9}{|c|}{$\begin{array}{c}\text { Obstetrics and } \\
\text { Gynecology }\end{array}$} \\
\hline & Number of interventions & 12 & 6 & 7 & 19 & 42 & 36 & 32 \\
\hline & Number of affected doses & 65 & 12 & 4 & 54 & 76 & 61 & 43 \\
\hline & Resistance rate & $0 \%$ & $50 \%$ & $71 \%$ & $21 \%$ & $14 \%$ & $6 \%$ & $13 \%$ \\
\hline \multicolumn{9}{|l|}{ Oncology } \\
\hline & Number of interventions & 8 & 18 & 4 & 6 & 6 & 2 & 2 \\
\hline & Number of affected doses & 48 & 34 & 22 & 22 & 19 & 3 & 10 \\
\hline & Resistance rate & $0 \%$ & $39 \%$ & $25 \%$ & $0 \%$ & $0 \%$ & $0 \%$ & $0 \%$ \\
\hline \multicolumn{9}{|c|}{ Opthalmology } \\
\hline & Number of interventions & 3 & 0 & 0 & 1 & 0 & 1 & 0 \\
\hline & Number of affected doses & 9 & 0 & 0 & 2 & 0 & 0 & 0 \\
\hline \multicolumn{9}{|l|}{ Orthopedics } \\
\hline & Number of interventions & 15 & 18 & 6 & 21 & 19 & 35 & 36 \\
\hline & Number of affected doses & 43 & 30 & 7 & 59 & 87 & 119 & 62 \\
\hline & Resistance rate & $13 \%$ & $50 \%$ & $33 \%$ & $24 \%$ & $26 \%$ & $20 \%$ & $3 \%$ \\
\hline
\end{tabular}




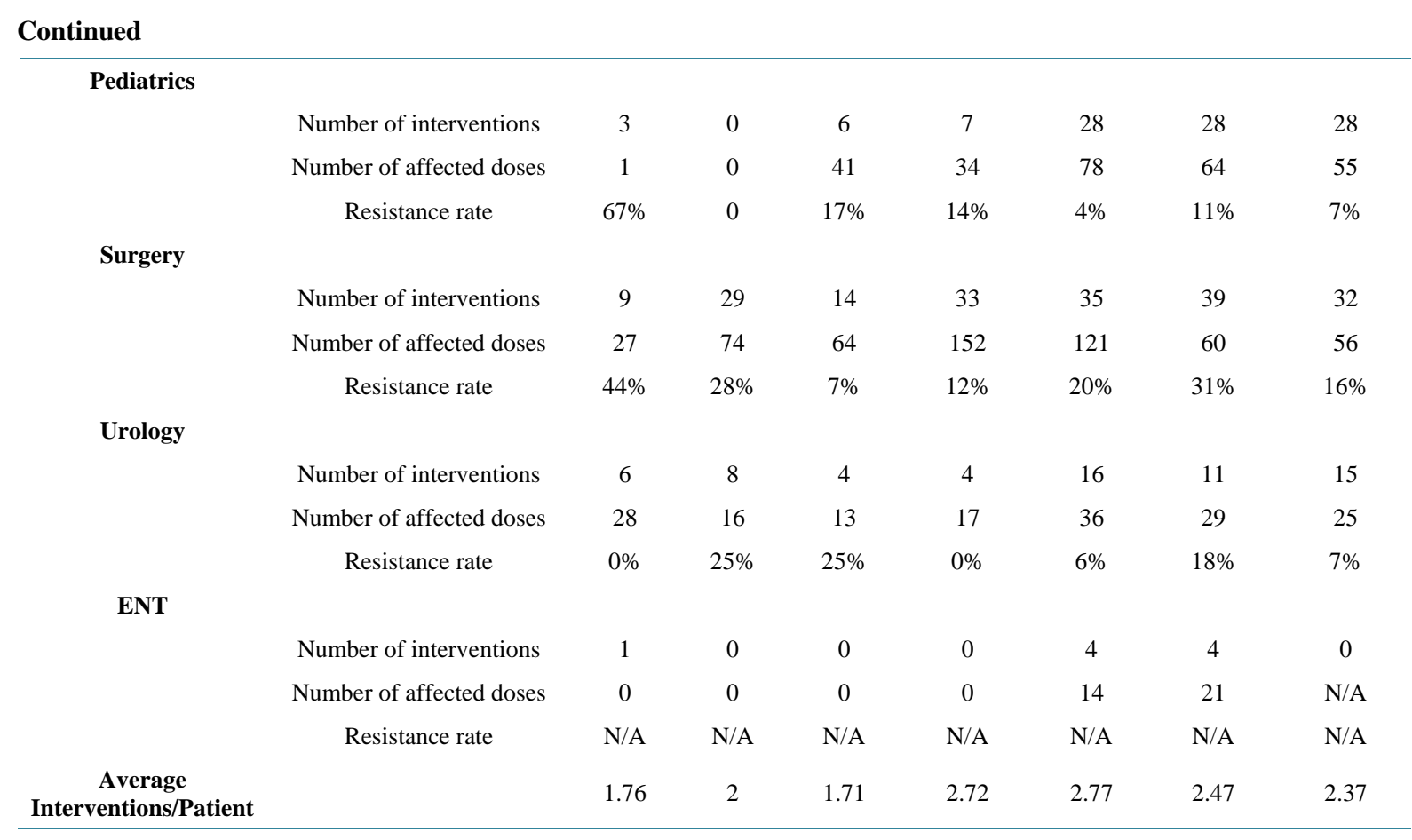

substantial patient safety challenges because of the fast-pace, complex problems, and the urgent high-risk decision-making by physicians with varying levels of critical care training, often with incomplete data [14]. Furthermore, critically ill patients may be particularly vulnerable to iatrogenic injury because of the severity and instability of their illness and their frequent need for high-risk interventions and medications [15]. Literature provides evidence that, pharmacist involvement improves patient safety [16] [17], where the presence of clinical pharmacists in pediatric ICU decreased serious medication problems by 79\% [18], and in adult ICU preventable adverse drug events were decreased by $66 \%$ [19].

Prescribing errors were the most frequently reported problems, possibly due to poor pharmacological background of the physicians, poor communication between the different healthcare providers and increased physician workload, fatigue and stress for physicians as reported in other studies [20]. These factors accounted for medication problems in other studies especially in the Middle East [21] [22]. As a result, a pharmacologically oriented physicians' training program was developed, and delivered and communication skills course for healthcare professionals was tailored to be delivered.

Drug administration is crucial in the medication management process since the possibilities of correcting errors at this stage are limited [23] [24]. Nursing drug administration errors were observed to be the second highest problem in this study, being mainly attributed to the large numbers of patients under the nurse's care where the ratio was 1:10, increasing both the work-load and the stress level. This nurse to patient ratio is much higher than those reported in other countries [25]. Nursing skills and training are other factors linked to such errors; the hospital had two nursing levels based on their educational background, the head nurses who work in morning shifts only, are university graduates and not in charge of patients. The second level is the charge nurses, who were graduates of two year nursing technical schools. All the administration errors could have been easily avoided by proper training of the nurses. Hence, a focused nursing training program was proposed by the pharmacy team to be routinely delivered to overcome the educational factor. Although nurses were encouraged to report administration errors; however, one deterring factor is the fear of losing their jobs or being subjected to disciplinary actions [22] [26], creating the need for constructive strategies to minimize such fears [27].

In the current study, investigators demonstrated how simple nurse instruction by pharmacists can lead to the correction of a substantial percentage of DRPs. The pharmacists' interventions, however, increased over the study period, probably due to the increase in the total number of audited doses and the increased experience of the pharmacists. The average resistance rate to pharmacist interventions was $21 \%$ compared to only $1 \%$ in one US study [19], and $20.8 \%$ in a French study [28]. Consultants could be more resistant to pharmacist interven- 
tions as was earlier demonstrated that physicians' age negatively correlated with attitude towards pharmacists' interventions [29] [30].

Medication problems may occur throughout the entire drug distribution system, from prescribing to administration [2]. Although, human errors are impossible to eliminate, the goal is to bring medication problems down to a minimum. Exploring the reasons behind these problems, and implementing efficient systems are the key processes to minimize human error. However, there has to be a system to stop carelessness and violations that are intentional rather than a human or system error in pursuit of patient safety [31]. To ensure patient safety, surveys of DRPs are indispensible since appropriate recommendations are dependent on the setting and background culture.

Additional contributing factors to DRPs could be miscommunications such as illegible medication orders, look-alike drug names, confusion of brand and generic names [32], interruptions or distractions during prescribing, dispensing and administration [33] [34] and the absence of extra checking procedures to further recover problems [35]. As a result of these findings, the pharmacy department in the hospital created a look-alike soundalike list. Also, a generic name prescribing policy was developed, as well as a reconciliation policy. In addition, medication auditing systems were started along with physician and nursing training programs including a pharmacology-oriented physicians' training program and a communication skills course for healthcare and a pharmacy and therapeutic committee (PTC) was created to be responsible for reporting, and discussing all the identified DRPs in the hospital under study.

Increasing the number of nurses could have minimized the number of administration errors. However, due to financial constraints, the nurse workload was quite difficult to be solved, but a focused nursing training program was proposed by the pharmacy team to be routinely delivered to overcome the educational factor. Nurses were also, encouraged to report administration errors.

Nevertheless, it should be noted that this was a single center study, pharmacists identifying DRPs worked 6 days per week for one morning shift only and there was no possibility of auditing all the doses nor of implementing a quality assurance system where medication orders could be re-checked for missed problems due to the limited number of pharmacists. A post discharge follow-up was also not possible. In addition, there was no possibility of comparing DRP's before and after implementation of the pharmacist medication review system due to the preceding lack of reporting DRP's by the hospital. Studies in other Egyptian hospitals need to be performed to further address drug related problems with interventions focusing actual outcomes, including quality of life, cost, morbidity and mortality, to address post-discharge follow-up and to record the impact of the medication review process on discharge process, on the primary care services the patient will be receiving, and on the estimate cost savings due to pharmacists interventions.

\section{Conclusion}

In conclusion, medication errors or problems within the Egyptian hospital were found common among hospitalized patients. The most common issues were prescribing errors, administration errors and over doses. Factors associated with an increased risk of such problems were physicians' resistance, shortage of nursing staff, and renal impairment. Clinical pharmacists trained in pharmacotherapy could help to detect problems/errors and reduce their effects, thus improving the quality of prescription, and patient's safety.

\section{Disclosure Statement}

The authors have no conflict of interest to report.

\section{Acknowledgments}

The authors would like to thank the all the pharmacy team in the study site for the technical support.

\section{References}

[1] Tully, M.P., Ashcroft, D.M., Dornan, T., Lewis, P.J., Taylor, D. and Wass, V. (2009) The Causes of and Factors Associated with Prescribing Errors in Hospital Inpatients: A Systematic Review. Drug Safety, 32, 819-836. http://dx.doi.org/10.2165/11316560-000000000-00000

[2] Van Den Bemt, P.M., Egberts, T.C., De Jong-Van Den Berg, L.T. and Brouwers, J.R. (2000) Drug-Related Problems 
in Hospitalised Patients. Drug Safety, 22, 321-333. http://dx.doi.org/10.2165/00002018-200022040-00005

[3] Shah, A. (2010) Pharmacy Intervention in the Medication Use. International Pharmaceutical Federation (FIP).

[4] Kohn, L., Corrigan, J. and Donaldson, M. (2000) To Err Is Human: Building a Safer Health System. Institute of Medicine, Washington DC.

[5] Alagha, H.Z., Badary, O.A., Ibrahim, H.M. and Sabri, N.A. (2011) Reducing Prescribing Errors in the Paediatric Intensive Care Unit: An Experience from Egypt. Acta Paediatrica, 100, e169-e174. http://dx.doi.org/10.1111/j.1651-2227.2011.02270.x

[6] Kandil, M., Sayyed, T., Emarh, M., Ellakwa, H. and Masood, A. (2012) Medication Errors in the Obstetrics Emergency Ward in a Low Resource Setting. Journal of Maternal-Fetal and Neonatal Medicine, 25, 1379-1382. http://dx.doi.org/10.3109/14767058.2011.636091

[7] Sabry, N.A., Farid, S.F. and Abdel Aziz, E.O. (2009) Role of the Pharmacist in Identification of Medication Related Problems in the Icu: A Preliminary Screening Study in an Egyptian Teaching Hospital. Australian Journal of Basic and Applied Sciences, 3, 995-1003.

[8] Strand, L.M., Morley, P.C., Cipolle, R.J., Ramsey, R. and Lamsam, G.D. (1990) Drug-Related Problems: Their Structure and Function. DICP, 24, 1093-1097.

[9] Anderson, A.C., Brodin, H. and Nilsson, J.L.G. (2003) Pharmacist Interventions in Relation to Patient Drug-Related Problems. Journal of Social and Administrative Pharmacy, 20, 82-91.

[10] U.S. Department of Health and Human Services (2012) Guidance for Industry and Investigators: Safety Reporting Requirements for Inds and Ba/Be Studies.

[11] Prot, S., Fontan, J.E., Alberti, C., Bourdon, O., Farnoux, C., Macher, M.A., Foureau, A., Faye, A., Beaufils, F., Gottot, S. and Brion, F. (2005) Drug Administration Errors and Their Determinants in Pediatric in-Patients. International Journal for Quality in Health Care, 17, 381-389. http://dx.doi.org/10.1093/intqhc/mzi066

[12] FDA (2012) Medwatch the Fda Safety Information and Adverse Event Reporting Program. http://www.fda.gov/Safety/MedWatch/HowToReport/ucm053087.htm

[13] Taegtmeyer, A.B., Curkovic, I., Corti, N., Rosen, C., Egbring, M., Russmann, S., Gantenbein, A.R., Weller, M. and Kullak-Ublick, G.A. (2012) Drug-Related Problems and Factors Influencing Acceptance of Clinical Pharmacologists Alerts in a Large Cohort of Neurology Inpatients. Swiss Medical Weekly, 142, 13615.

[14] Beckmann, U., Bohringer, C., Carless, R., Gillies, D.M., Runciman, W.B., Wu, A.W. and Pronovost, P. (2003) Evaluation of Two Methods for Quality Improvement in Intensive Care: Facilitated Incident Monitoring and Retrospective Medical Chart Review. Critical Care Medicine, 31, 1006-1011. http://dx.doi.org/10.1097/01.CCM.0000060016.21525.3C

[15] Cullen, D.J., Sweitzer, B.J., Bates, D.W., Burdick, E., Edmondson, A. and Leape, L.L. (1997) Preventable Adverse Drug Events in Hospitalized Patients: A Comparative Study of Intensive Care and General Care Units. Critical Care Medicine, 25, 1289-1297. http://dx.doi.org/10.1097/00003246-199708000-00014

[16] Lu, C.Y., Ross-Degnan, D., Soumerai, S.B. and Pearson, S.A. (2008) Interventions Designed to Improve the Quality and Efficiency of Medication Use in Managed Care: A Critical Review of the Literature-2001-2007. BMC Health Services Research, 8, 75. http://dx.doi.org/10.1186/1472-6963-8-75

[17] Klopotowska, J.E., Kuiper, R., van Kan, H.J., de Pont, A.C., Dijkgraaf, M.G., Lie, A.H.L., Vroom, M.B. and Smorenburg, S.M. (2010) On-Ward Participation of a Hospital Pharmacist in a Dutch Intensive Care Unit Reduces Prescribing Errors and Related Patient Harm: An Intervention Study. Critical Care, 14, R174. http://dx.doi.org/10.1186/cc9278

[18] Kaushal, R., Bates, D.W., Abramson, E.L., Soukup, J.R. and Goldmann, D.A. (2008) Unit-Based Clinical Pharmacists' Prevention of Serious Medication Errors in Pediatric Inpatients. American Journal of Health, System Pharmacy, 65, 1254-1260. http://dx.doi.org/10.2146/ajhp070522

[19] Leape, L.L., Cullen, D.J., Clapp, M.D., Burdick, E., Demonaco, H.J., Erickson, J.I. and Bates, D.W. (1999) Pharmacist Participation on Physician Rounds and Adverse Drug Events in the Intensive Care Unit. JAMA, 282, 267-270. http://dx.doi.org/10.1001/jama.282.3.267

[20] Tully, M.P. and Buchan, I.E. (2009) Prescribing Errors during Hospital Inpatient Care: Factors Influencing Identification by Pharmacists. Pharmacy World \& Science, 31, 682-688. http://dx.doi.org/10.1007/s11096-009-9332-x

[21] Alsulami, Z., Conroy, S. and Choonara, I. (2013) Medication Errors in the Middle East Countries: A Systematic Review of the Literature. European Journal of Clinical Pharmacology, 69, 995-1008. http://dx.doi.org/10.1007/s00228-012-1435-y

[22] Unver, V., Tastan, S. and Akbayrak, N. (2012) Medication Errors: Perspectives of Newly Graduated and Experienced Nurses. International Journal of Nursing Practice, 18, 317-324. http://dx.doi.org/10.1111/j.1440-172X.2012.02052.X

[23] Van Den Bemt, P.M., Robertz, R., De Jong, A.L., Van Roon, E.N. and Leufkens, H.G. (2007) Drug Administration 
Errors in an Institution for Individuals with Intellectual Disability: An Observational Study. Journal of Intellectual Disability Research, 51, 528-536.

[24] Wright, A.A. and Katz, I.T. (2005) Bar Coding for Patient Safety. The New England Journal of Medicine, 353, 329331. http://dx.doi.org/10.1056/NEJMp058101

[25] Welton, J.M. (2007) Mandatory Hospital Nurse to Patient Staffing Ratios: Time to Take a Different Approach. The Online Journal of Issues in Nursing, 12, Published Online.

[26] Mrayyan, M.T. and Al-Atiyyat, N. (2011) Medication Errors in University-Affiliated Teaching Hospitals as Compared to Non-University-Affiliated Teaching Hospitals in Jordan. Nursing Forum, 46, 206-217. http://dx.doi.org/10.1111/j.1744-6198.2011.00241.x

[27] Kim, K.S., Kwon, S.H., Kim, J.A. and Cho, S. (2011) Nurses' Perceptions of Medication Errors and Their Contributing Factors in South Korea. Journal of Nursing Management, 19, 346-353. http://dx.doi.org/10.1111/j.1365-2834.2011.01249.x

[28] Bedouch, P., Tessier, A., Baudrant, M., Labarere, J., Foroni, L., Calop, J., Bosson, J.L. and Allenet, B. (2011) Computerized Physician Order Entry System Combined with On-Ward Pharmacist: Analysis of Pharmacists' Interventions. Journal of Evaluation in Clinical Practice, 18, 911-918. http://dx.doi.org/10.1111/j.1365-2753.2011.01704.x

[29] Haxby, D.G., Weart, C.W. and Goodman Jr., B.W. (1988) Family Practice Physicians' Perceptions of the Usefulness of Drug Therapy Recommendations from Clinical Pharmacists. American Journal of Hospital Pharmacy, 45, 824-827.

[30] Bradshaw, S.J. and Doucette, W.R. (1998) Community Pharmacists as Patient Advocates: Physician Attitudes. Journal of the American Pharmaceutical Association (Wash), 38, 598-602.

[31] Merry, A.F. and Anderson, B.J. (2011) Medication Errors-New Approaches to Prevention. Pediatric Anesthesia, 21, 743-753. http://dx.doi.org/10.1111/j.1460-9592.2011.03589.x

[32] Benjamin, D.M. (2003) Reducing Medication Errors and Increasing Patient Safety: Case Studies in Clinical Pharmacology. The Journal of Clinical Pharmacology, 43, 768-783. http://dx.doi.org/10.1177/0091270003254794

[33] Leape, L.L., Bates, D.W., Cullen, D.J., Cooper, J., Demonaco, H.J., Gallivan, T., Hallisey, R., Ives, J., Laird, N., Laffel, G., et al. (1995) Systems Analysis of Adverse Drug Events. Ade Prevention Study Group. JAMA, 274, 35-43. http://dx.doi.org/10.1001/jama.1995.03530010049034

[34] Gladstone, J. (1995) Drug Administration Errors: A Study into the Factors Underlying the Occurrence and Reporting of Drug Errors in a District General Hospital. Journal of Advanced Nursing, 22, 628-637. http://dx.doi.org/10.1046/j.1365-2648.1995.22040628.x

[35] Cohen, M.R., Blanchard, N., Fredrico, F., Magelli, M., Lomax, C., Greiner, G. and Poole, R.L. (1998) Draft Guidelines for Preventing Medication Errors in Pediatrics. Journal of Pediatric Pharmacy Practice, 3, 189-202. 